\title{
Lung Ultrasonography to Diagnose Bronchopulmonary Dysplasia of Premature Infants
}

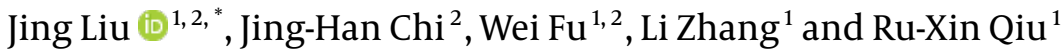 \\ ${ }^{1}$ Department of Neonatology and NICU, Beijing Chaoyang District Maternal and Child Healthcare Hospital, Beijing, China \\ ${ }^{2}$ Department of Neonatology and NICU, Bayi Children's Hospital Affiliated to the Seventh Medical Center of Chinese PLA General Hospital, Beijing, China \\ "Corresponding author: Department of Neonatology and NICU, Beijing Chaoyang District Maternal and Child Healthcare Hospital, Beijing, China. Email: liujingbj@live.cn
}

Received 2020 September 20; Revised 2021 May 07; Accepted 2021 May 09.

\begin{abstract}
Background: Bronchopulmonary dysplasia (BPD) is a common severe respiratory problem in premature infants, and imaging information has important reference value for its diagnosis. Recently, lung ultrasonography (LUS) has been successfully used for the diagnosis and differential diagnosis of neonatal lung diseases (NLDs), but the study of the diagnosis of BPD is still rare.

Objectives: The purpose of this study was to investigate the ultrasonographic characteristics of BPD and its value for the diagnosis and differential diagnosis of premature infants' BPD.

Methods: From January 2015 to December 2019, 25 premature infants diagnosed with early-stage BPD and 32 infants diagnosed with late-stage BPD according to their medical history, clinical manifestation, and chest X-ray were included in this study. The LUS examinations were performed on each infant. The LUS findings were recorded and compared with those of 40 premature infants without lung diseases.

Results: The gestational age of 25 early-stage BPD infants was $26^{+1}-31^{+6}$ weeks, and their birth weight was between 730 and $1,810 \mathrm{~g}$. The gestational age of 32 late-stage BPD infants was 26 - 32 weeks, and their birth weight was 750-1,760 g. The gestational age of 40 control infants was $25^{+6}-32^{+1}$ weeks, and their birth weight was $810-2,050 \mathrm{~g}$. There was no difference in the proportion of primary lung diseases (including RDS, TTN, pneumonia, etc.) between the three groups. The proportions of infants receiving invasive and/or noninvasive respiratory support at admission in the three groups of early BPD, late BPD, and normal control were 20/25 (80.0\%), 26/32 (81.2\%), and 33/40 (77.5\%), respectively, with no significant difference $(\mathrm{P}>0.05)$. The mechanical ventilation duration over one week in three groups was $15 / 20$ (75\%), 21/26 (80.7\%), and 24/33 (72.7\%), respectively, with no significant difference $(\mathrm{P}>0.05)$. Nonspecific pleural line abnormalities were seen in all early and late BPD patients (100\%), alveolar-interstitial syndrome (AIS) in 16 cases (64\%) of early BPD and 32 cases of late BPD infants (100\%), pleural insect erosion-like change (PIE-like change) in two cases of early-stage BPD infants (8.0\%) and 20 cases (62.5\%) of late-stage BPD infants, and air vesicle signs (AVS) only in 17 cases of late-stage BPD infants. The sensitivity and specificity of PIE-like change for the diagnosis of late-stage BPD were $62.5 \%$ and $92.0 \%$, respectively, and the sensitivity and specificity of AVS for the diagnosis of late-stage BPD were 53.1 and $100 \%$, respectively.

Conclusions: Lung ultrasonography is not specific for the diagnosis of early-stage BPD, but has a high reference value and specificity for the diagnosis of late-stage BPD when combined with obvious pulmonary fibrosis and pulmonary vesicle formation, which is mainly manifested by AIS, PIE-like change, and AVS.
\end{abstract}

Keywords: Bronchopulmonary Dysplasia (BPD), Premature Infants, Lung Ultrasonography (LUS), Alveolar-Interstitial Syndrome (AIS), Pleural Insect Erosion-Like Change (PIE-Like Change), Air Vesicle Signs (AVS)

\section{Background}

Bronchopulmonary dysplasia (BPD) is known as a chronic lung disease (CLD), which is a common severe respiratory problem in premature infants, especially extremely premature infants. Besides, BPD seriously affects the survival rate of premature infants and their quality of life. For more than 50 years, medical staff has carried out many systemic studies on the physiological mechanism, prevention, and control measures, as well as lung develop- ment, damage, and repair mechanisms in premature infants. Although important progress has been made, the incidence rate of BPD has not significantly decreased. For example, in extremely premature infants with gestational age $<29$ weeks, the incidence of BPD is still as high as $43 \%$. These survivors also face a large risk of early death, and they need a tracheosto-my, long-term mechanical ventilation support, and even are at risk of lifelong respiratory system dis-ease (1). Early, timely, and effective means of detec- 
tion and diagnosis can provide reliable reference indices for early prevention and diagnosis, which is of great significance for reducing the incidence, alleviating the severity, and improving the prognosis of BPD.

The diagnosis of BPD mainly depends on clinical manifestations, but pulmonary imaging exami-nation (including chest X-ray (CXR) and CT) has always played an important role in its diagnosis $(2,3)$. In recent years, the use of lung ultrasonography (LUS) to diagnose neonatal lung diseases (NLDs) has been widely accepted and recognized by clinicians and sonographers. Compared with traditional CXR, LUS has higher accuracy, reliability, and specificity in diagnosing NLDs (4-6); therefore, LUS has been widely carried out (7-9). Even in some well-developed neonatal intensive care units (NICUs), LUS has replaced CXR examination for routine use in the diagnosis of $\operatorname{NLDs}(4,9)$. Re-search on the physiological mechanism of BPD has not shown consistent results, and the diagnostic criteria are not unified $(10,11)$. A few authors believe that LUS can predict the occurrence of BPD (11-13); however, research on the use of LUS to diagnose BPD has not been reported.

From January 2015 to December 2019, 25 early-stage BPD and 32 late-stage BPD patients were examined by LUS to preliminarily explore the feasibility of diagnosing BPD by ultrasound.

\section{Methods}

\subsection{Patients}

The institutional review board of Beijing Chaoyang District Maternal and Child Healthcare Hospital approved the study protocol (No. 2011-LC-Ped-01). Informed consent was obtained from their guardians who were involved in this study. This prospective study included 25 early-stage BPD and 32 late-stage BPD infants who were hospitalized in the NICU of the Bayi Children's Hospital and Beijing Chaoyang District Maternal and Child Healthcare Hospital from January 2015 to December 2019. The clinical data were collected by different doctors, and the ultrasound operator was blinded to the clinical condition of the BPD infants. During the study period, 40 preterm infants without NLDs were randomly selected as controls.

\subsection{BPD Diagnostic Criteria}

The criteria used to diagnose BPD in this study were in line with the definition developed by the National Institutes of Health (NIH). Thus, BPD referred to the requirement of oxygen support ( $>21 \%$ ) for at least 28 days (14). Regarding early-stage or late-stage BPD, we used the following criteria in this study: (1) early-stage BPD meant that there was no fibrosis or vesicle formation on CXR or CT scan; while (2) late-stage BPD meant that there was significant fibrosis or vesicle formation on CXR or CT scan.

\subsection{LUS Examination \\ 2.3.1. Instruments}

The instruments used in this study were GE Voluson S10 or Philip EP5 ultrasound equipment, which is being used in our NICU. The frequency of the linear array probe was 10 to $18 \mathrm{MHz}$. All operators had been uniformly trained to be proficient in LUS.

\subsubsection{Examination Method}

The LUS examinations were performed by systematically trained neonatologists. In a quiet state, the infants were placed in the supine, lateral, or prone positions for the scan. Each lung was divided into three regions of anterior, lateral, and posterior by the anterior and posterior axillary lines. The probe was perpendicular to the ribs. Each region of both lungs was scanned carefully $(15,16)$.

\subsubsection{Observation Indexes}

The observation indices included A-line, B-line, confluent B-lines, compact B-lines, alveolar-interstitial syndrome (AIS), pleural line, lung sliding, and lung pulse. The exact definitions of the terms used have been previously described in detail (15-18).

\subsubsection{Statistical Analysis}

Statistical Package for the Social Sciences (SPSS) 19.0 software was used to statistically analyze the data. Fisher's exact test was used to compare the positive rates of the LUS test results of newborns from each group. These results were used to calculate the specificity and sensitivity of the main test results for the diagnosis of BPD. A value of $\mathrm{P}<$ 0.05 indicated statistically significant differences. The information reported was evaluated by an expert.

\section{Results}

\subsection{General Information}

The gestational age of 25 early-stage BPD infants was $26^{+1}-31^{+6}$ weeks, and their birth weight was be-tween 730 and 1,810 g. The gestational age of 32 late-stage BPD infants was 26 - 32 weeks, and their birth weight was between 750 and $1,760 \mathrm{~g}$. The gestational age of 40 control infants was $25^{+6}-32^{+1}$ weeks, and their birth weight was between 810 and 2,050 g. There were no differences in the proportion of their primary lung diseases (including RDS, TTN, pneumonia, etc.) between the three groups. The proportions of infants receiving invasive and/or noninvasive respiratory support at admission in the three groups of earlystage BPD, late-stage BPD, and normal controls were 20/25 
(80.0\%), 26/32 (81.2\%), and 33/40 (77.5\%), respectively, with no significant differences $(\mathrm{P}>0.05)$. The mechanical ventilation duration over one week in the early-stage BPD, latestage BPD, and control groups was 15/20 (75\%), 21/26 (80.7\%), and $24 / 33$ (72.7\%), respectively, with no significant differences $(\mathrm{P}>0.05)$.

\subsection{Normal Lung LUS Pattern of Manifestation}

Briefly, normal neonatal lung imaging showed a 'bamboo sign' under B-mode ultrasonography (15-18). Pleural lines and A-lines were smooth, regular, and straight hyperechoic lines, parallel and equidistant from each other. Alines gradually diminished and finally disappeared off the screen (Figure 1).

\subsection{LUS findings in BPD Patients}

The major LUS findings in BPD infants were as follows: (1) nonspecific pleural line abnormalities, including coarse or fuzzy, which were seen in all early- and late-stage BPD patients (100\%); (2) pleural insect erosion (PIE)-like changes, one kind of specific pleural line abnormalities, that were mild consolidations and non-consolidated lesions alternate at the pleural line, resulting in the discontinuity of the thickened pleural line, which looked like the bites of insects on ultrasound. The PIE-like changes were found in two early-stage BPD infants (8.0\%) and 20 (62.5\%) late-stage BPD infants; (3) AIS, which was observed in 16 early-BPD infants (64\%) and 32 late-BPD infants (100\%) (Figure 2); (4) Air Vesicle Signs (AVS), which were dot-like hyperechoic patterns situated in the fields of AIS. We name this pattern AVS in this paper. Air vesicle signs were seen only in 17 late-stage BPD infants (53.1\%), while they were not seen in early-stage BPD infants (Figure 3).

\subsection{Sensitivity and Specificity of LUS in Diagnosing BPD}

When reviewing the published literature and our research on neonatal lung diseases, we found no PIE-like changes or AVS in other kinds of lung diseases, including pneumonia, atelectasis, lung bleeding, pulmonary edema, pneumothorax, and normal lungs (4-7, 16-28). Besides, PIElike changes or AVS were only seen in late-stage BPD patients. Thus, we believe that PIE-like changes and AVS are specific signs of late-stage BPD observed on LUS. Therefore, the sensitivity and specificity of PIE-like changes for the diagnosis of late-stage BPD were $62.5 \%$ and $92.0 \%$, respectively, and the sensitivity and specificity of AVS for the diagnosis of late-stage BPD were $53.1 \%$ and $100 \%$, respectively.

\section{Discussion}

\subsection{Long-term Oxygen Dependence in Premature Infants is not Equivalent to $B P D$}

Previously, we conducted LUS examinations on 50 premature infants who had been oxygen-dependent for more than two months. The results showed that $36 \%$ of them had other kinds of lung diseases, including atelectasis, pneumonia, severe lung edema (possibly due to cardiac reasons, fluid replacement reasons, etc.), and lung edema with focal lung consolidation (29). When appropriate intervention measures were taken to resolve the above reasons, the oxygen dependence of some infants disappeared completely, and the degree of oxygen dependence in some infants was significantly reduced. Therefore, these infants would not be readily diagnosed as having BPD. Patients whose oxygen dependence disappears would not probably have BPD, and those whose oxygen dependence is mitigated may have different degrees of BPD; and although their initial need for oxygen may be due to lung inflammation, atelectasis, or serious diseases such as lung edema, the original X-ray may not find existing lung lesions. However, LUS is still not common, and there is no dynamic X-ray or further chest CT examination, thus making these patients receive long-term oxygen therapy, resulting in BPD or oxygen dependence. We refer to this type of BPD due to insufficient knowledge or improper clinical management as "iatrogenic BPD" (29). Besides, LUS can make a clearer early diagnosis of the cause of long-term oxygen dependence in preterm infants. Because atelectasis, pneumonia, or lung edema should not be the pathological changes of BPD itself, they may be BPD complications, comorbidities, or even the reasons for BPD. Therefore, if LUS examination reveals atelectasis, pneumonia, or other pathological changes of the lungs in longterm oxygen-dependent infants, the patient should not be quickly diagnosed as having BPD.

\subsection{LUS Diagnostic Value on BPD}

According to our preliminary observations and research results, there are no specific changes in ultra-sound imaging in early-stage BPD. When pathological changes such as significant pulmonary fibrosis and vascularization appear in late-stage BPD, when abnormalities such as pulmonary edema, thoracic dysplasia, atelectasis, pneumonia, heart diseases, and upper airway disease (such as softening of the trachea) are excluded, and when the following signs appear on ultrasound imaging, the possibility of BPD should be considered. The signs include: (1) AIS, which is the most common LUS manifestation of early-and late-stage BPD, but it is not an indicative sign because it can also be seen in many other lung diseases [17-28]. The cause may be related to pulmonary fibrosis or increased 


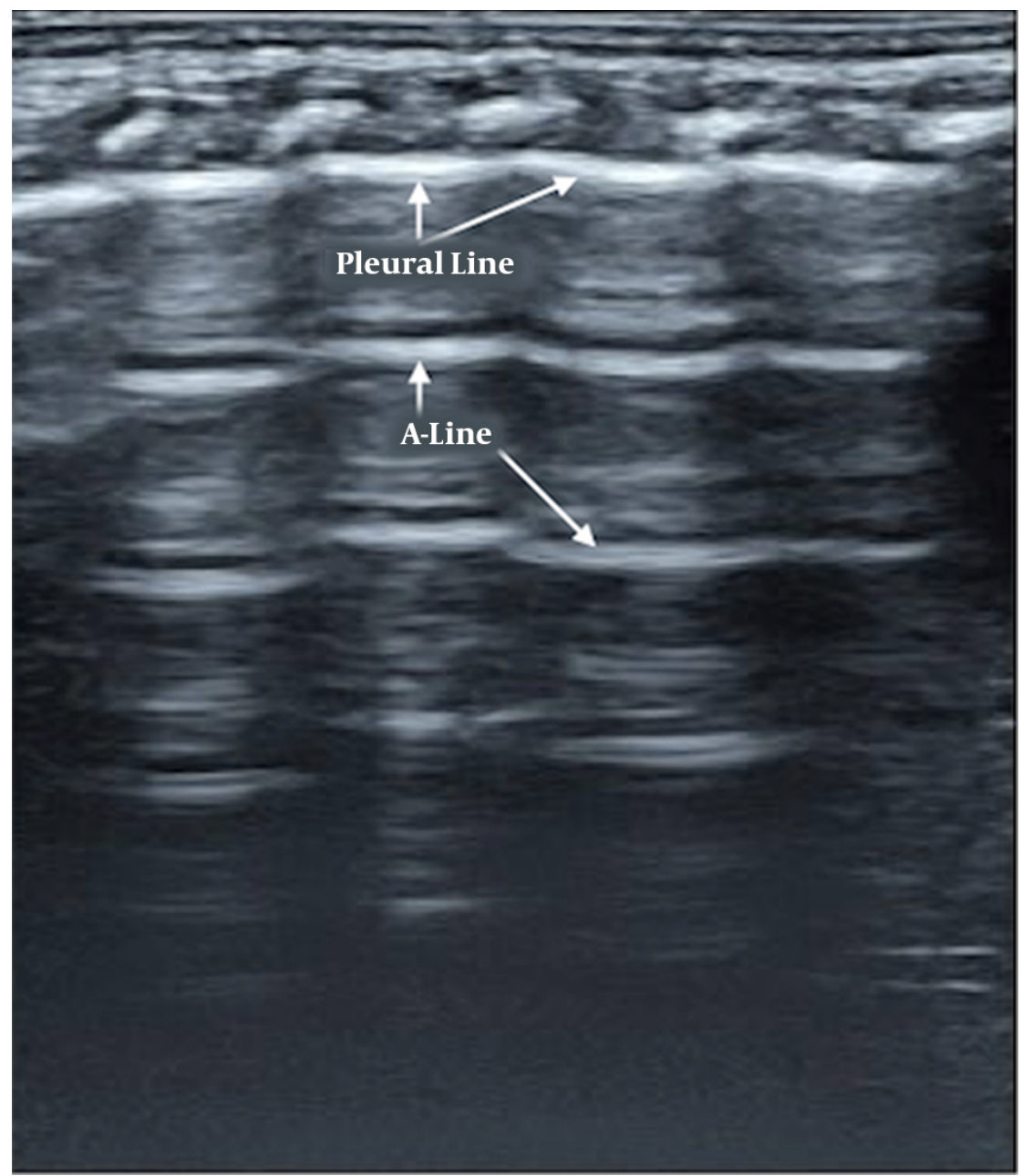

Figure 1. Normal lung ultrasound manifestation. Pleural lines and A-lines are smooth, regular, and straight hyperechoic lines, parallel and equidistant from each other. A-lines gradually diminish and finally disappear off the screen, which formed a manifestation of bamboo-like, named as "bamboo sign".

water content in the lung tissue; (2) nonspecific pleural line abnormality, including coarse or fuzzy is one common ultrasound finding in BPD patients; (3) specific pleural line abnormalities that are PIE-like changes, and one specific ultrasound sign of late-stage BPD; the sensitivity and specificity of PIE-like changes for the diagnosis of latestage BPD were $62.5 \%$ and $92.0 \%$, respectively (Figure 2 ). The reason for this finding may be related to pulmonary fibrous tissue and capillary proliferation and degeneration in-volving the pleura, causing damage; (4) AVS, which indicates that the lung tissue has vesicle formation and air exists within it (Figure 3). The AVS is an ultrasound imaging sign that occurs in late-stage BPD. It shows scattered higher echo reflections on the ultrasound image, which is often located in the AIS fields (it should be distinguished from air bronchograms, which are located in consolidated lung areas). The AVS may correspond to pulmonary vesicles or pulmonary cysts found on chest X-rays or CT scans in BPD patients. This needs to be further studied and verified. The AVS is another specific ultrasound sign of late-stage BPD and had sensitivity and specificity of 53.1 and 100\%, respectively. Generally, these findings are diffusely in all lung fields, but they are easy to be found at the back of the chest, which may be related to more severe lesions in the back. However, it is not easy to distinguish VAS from bronchograms for those who are inexperienced in the diagnosis of BPD by ultrasound. According to our study and knowledge, the VAS exists within the vesicle-forming lung field while air bronchograms exist within consolidated lung tissue. Therefore, whether there is consolidation is the key to distinguish them. 

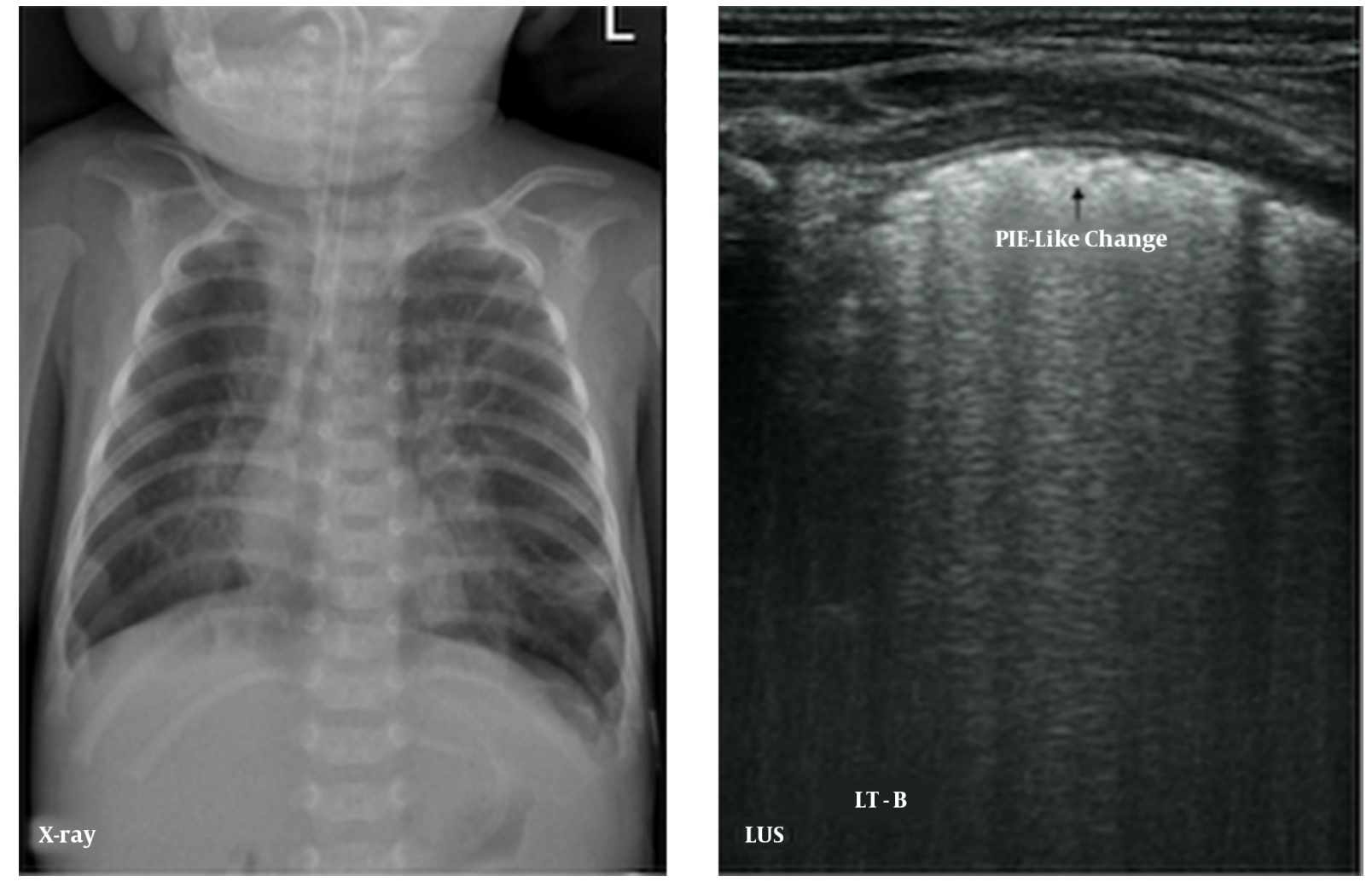

Figure 2. Pleural line insect erosion (PIE)-like change. LUS findings of pneumonia demonstrated in an oxygen-dependent extremely premature infant with a gestational age of 27 weeks at 102 days of birth. CXR showed bilateral lung texture increased, fuzzy and disorder, coarse interstitial opacification, especially the inner band of double lung fields, which is consistent with pulmonary tis-sue fibrosis. LUS shows the pleural line thickening, fuzzy, PIE-like change, and AIS, which is consistent with pulmonary fibrosis.

\subsection{Conclusion}

In conclusion, this paper is a preliminary study on the possibility of using ultrasound to diagnose BPD and its ultrasound imaging characteristics. The results showed that it is difficult to use LUS to diagnose early-stage BPD. Compared to the diagnosis of early-stage BPD, LUS has a higher value in the diagnosis of late-stage BPD when combined with obvious pulmonary fibrosis and pulmonary vesicle formation, which is mainly manifested by AIS, PIElike changes, and AVS. Among these characteristics, AIS is the most common ultrasound manifestation of BPD, while PIE-like changes and AVS are specific to diagnose late-stage BPD.

\subsection{Limitations}

Because the current understanding of BPD is not complete, diagnostic standards for BPD are not uniform. This paper presenting research on BPD diagnosis by ultrasound is also not sufficiently comprehensive. An in-depth understanding of BPD and the standardization of the diagnosis will facilitate further study of the value of ultrasound and ultrasound image characteristics for the diagnosis of BPD. Another limitation of this study is that we did not compare it with necrotizing pneumonia, which is also relatively rare in newborns. However, they should have different medical histories and clinical manifestations, and their differences in ultrasound images should be studied later.

\section{Footnotes}

Authors' Contribution: Jing Liu contributed to the study conception, lung ultrasound examination, data analysis, manuscript preparation, writing, and approval of the manuscript. Han-Jing Chi contributed to lung ultrasound examination, data analysis, manuscript preparation, baby care, and approval of the final manuscript. Wei Fu contributed to data analysis, manuscript preparation, and approval of the final manuscript. Li Zhang and Ru-Xin Qiu contributed to data analysis, manuscript preparation, and approval of the final manuscript.

Conflict of Interests: The authors declare that they have no competing interests. 

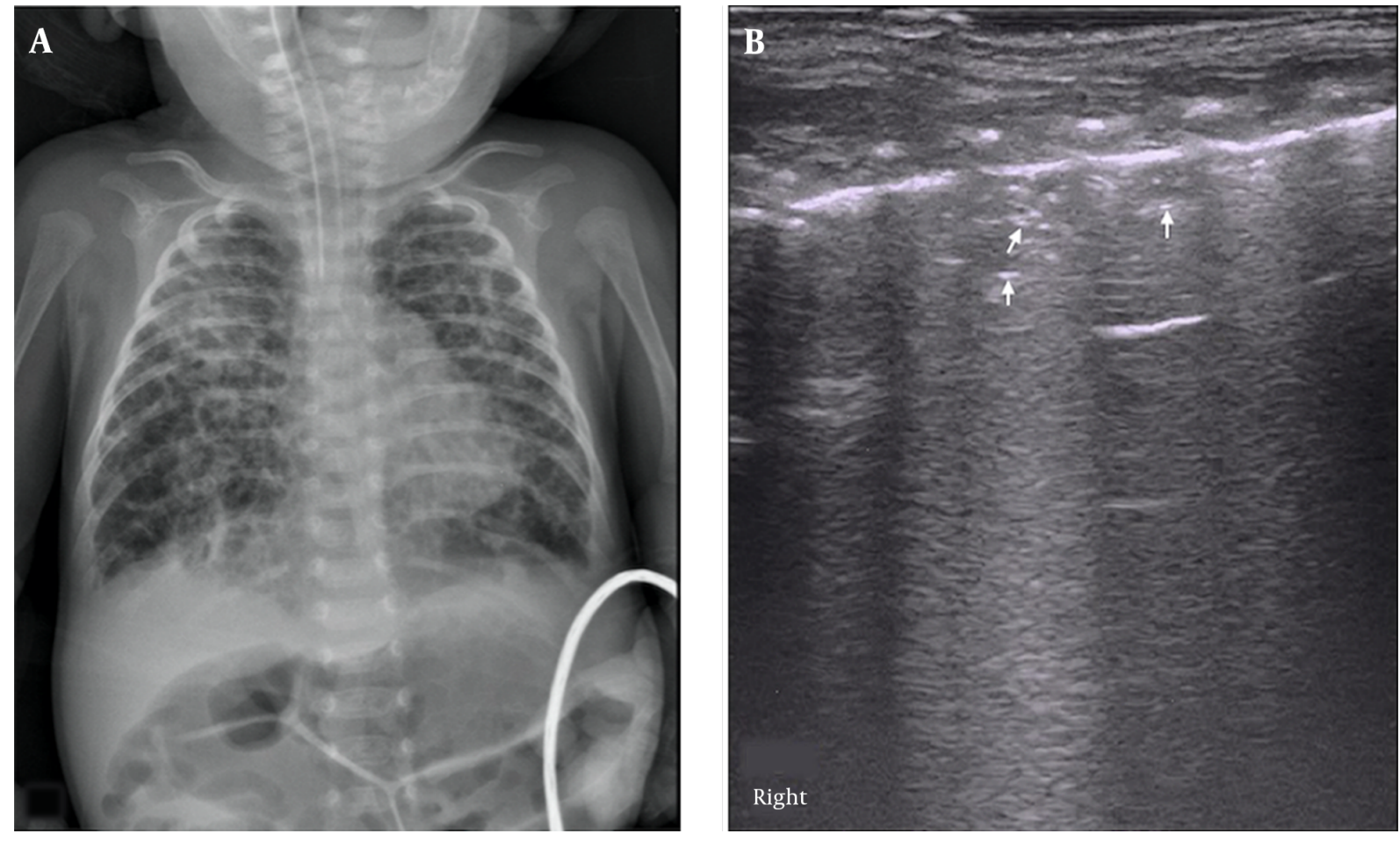

Figure 3. Air Vesicle Signs (AVS). LUS findings in an oxygen-dependent extremely premature infant with a gestational age of 28 weeks at four months of birth. CXR showed honeycomb lucency suggesting pulmonary vesicle formation (A). LUS showed pleural line thickening and fuzzy, scattered dot or linear hyperechoic reflection (arrow) is seen within the lung field, which is AVS (B).

Ethical Approval: The institutional review board of Beijing Chaoyang District Maternal and Child Healthcare Hospital approved the study protocol (No. 2011-LC-Ped-01).

Funding/Support: This work was supported by the Social Development Projects, Beijing Chaoyang District Bureau of Science, Technology, and Information (CYSF1922).

Informed Consent: Informed consent was obtained from the patients' guardians who were involved in this study.

\section{References}

1. Abman SH. Medical progress series: Bronchopulmonary dysplasia at 50 years-an introduction. J Pediatr. 2017;188:18-18 e1. doi: 10.1016/j.jpeds.2017.07.004. [PubMed: 28843303].

2. Semple T, Akhtar MR, Owens CM. Imaging bronchopulmonary dysplasia-a multimodality update. Front Med (Lausanne). 2017;4:88. doi: 10.3389/fmed.2017.00088. [PubMed: 28725645]. [PubMed Central: PMC5497953].

3. Li R, Zhang J. Diagnostic value of chest $\mathrm{CT}$ combined with $\mathrm{x}$-ray for premature infants with bronchopulmonary dysplasia. Medicine (Baltimore). 2018;97(9). e9723. doi: 10.1097/MD.0000000000009723. [PubMed: 29489675]. [PubMed Central: PMC5851751].

4. Liu J, Lovrenski J, Ye Hlaing A, Kurepa D. Neonatal lung diseases: Lung ultrasound or chest X-ray. J Matern Fetal Neonatal Med. 2021;34(7):117782. doi: 10.1080/14767058.2019.1623198. [PubMed: 31220971].

5. Alrajab S, Youssef AM, Akkus NI, Caldito G. Pleural ultrasonography versus chest radiography for the diagnosis of pneumothorax: Review of the literature and meta-analysis. Crit Care. 2013;17(5):R208. doi: 10.1186/cc13016. [PubMed: 24060427]. [PubMed Central: PMC4057340]

6. Hiles M, Culpan AM, Watts C, Munyombwe T, Wolstenhulme S. Neonatal respiratory distress syndrome: Chest X-ray or lung ultrasound? A systematic review. Ultrasound. 2017;25(2):80-91. doi: 10.1177/1742271X16689374. [PubMed: 28567102]. [PubMed Central: PMC5438053].

7. Elsayed YN. Lung ultrasound as a new technique for diagnosis of neonatal respiratory diseases. Neonatal Netw. 2018;37(4):224-32. doi: 10.1891/0730-0832.37.4.224. [PubMed: 30567920].

8. Chen SW, Fu W, Liu J, Wang Y. Routine application of lung ultrasonography in the neonatal intensive care unit. Medicine (Baltimore) 2017;96(2). e5826. doi: 10.1097/MD.0000000000005826. [PubMed: 28079811]. [PubMed Central: PMC5266173].

9. Gao YQ, Qiu RX, Liu J, Zhang L, Ren XL, Qin SJ. Lung ultrasound completely replaced chest X-ray for diagnosing neonatal lung diseases: A 3-year clinical practice report from a neonatal intensive care unit in China. J Matern Fetal Neonatal Med. 2020:1-8. doi: 10.1080/14767058.2020.1830369. [PubMed: 33032479].

10. Poindexter BB, Feng R, Schmidt B, Aschner JL, Ballard RA, Hamvas $\mathrm{A}$, et al. Comparisons and limitations of current definitions of bronchopulmonary dysplasia for the prematurity and respiratory outcomes program. Ann Am Thorac Soc. 2015;12(12):1822-30. doi 10.1513/AnnalsATS.201504-2180C. [PubMed: 26397992]. [PubMed Central: PMC4722827].

11. Gomez Pomar E, Concina VA, Samide A, Westgate PM, Bada HS Bronchopulmonary dysplasia: Comparison between the two most used diagnostic criteria. Front Pediatr. 2018;6:397. doi 
10.3389/fped.2018.00397. [PubMed: 30619792]. [PubMed Central: PMC6299117].

12. Pieper $\mathrm{CH}$, Smith J, Brand EJ. The value of ultrasound examination of the lungs in predicting bronchopulmonary dysplasia. Pediatr Radiol.2004;34(3):227-31. doi:10.1007/s00247-003-1102-7. [PubMed: 14685792].

13. Alonso-Ojembarrena A, Lubian-Lopez SP. Lung ultrasound score as early predictor of bronchopulmonary dysplasia in very low birth weight infants. Pediatr Pulmonol. 2019;54(9):1404-9. doi: 10.1002/ppul.24410. [PubMed: 31216121].

14. Abdelmawla M, Louis D, Narvey M, Elsayed Y. A Lung Ultrasound Severity Score predicts chronic lung disease in preterm infants. Am J Perinatol. 2019;36(13):1357-61. doi: 10.1055/s-0038-1676975. [PubMed: 30609427].

15. Jobe AH, Bancalari E. Bronchopulmonary dysplasia. Am J Respir Crit Care Med. 2001;163(7):1723-9. doi: 10.1164/ajrccm.163.7.2011060. [PubMed: 11401896].

16. Liu J. Lung ultrasonography for the diagnosis of neonatal lung disease. J Matern Fetal Neonatal Med. 2014;27(8):856-61. doi: 10.3109/14767058.2013.844125. [PubMed: 24028601].

17. Liu J, Copetti R, Sorantin E, Lovrenski J, Rodriguez-Fanjul J, Kurepa $D$, et al. Protocol and guidelines for point-of-care lung ultrasound in diagnosing neonatal pulmonary diseases based on international expert consensus. J Vis Exp. 2019;(145). doi: 10.3791/58990. [PubMed: 30907892].

18. Volpicelli G, Elbarbary M, Blaivas M, Lichtenstein DA, Mathis G, Kirkpatrick AW, et al. International evidence-based recommendations for point-of-care lung ultrasound. Intensive Care Med. 2012;38(4):577-91. doi: 10.1007/s00134-012-2513-4. [PubMed: 22392031].

19. Yousef $\mathrm{N}$. [Lung ultrasound in the newborn]. Arch Pediatr. 2016;23(3):317-21. French. doi: 10.1016/j.arcped.2015.12.001.

20. Kurepa D, Zaghloul N, Watkins L, Liu J. Neonatal lung ultrasound exam guidelines. J Perinatol. 2018;38(1):11-22. doi: 10.1038/jp.2017.140. [PubMed: 29144490].
21. Liu J, Chi JH, Ren XL, Li J, Chen YJ, Lu ZL, et al. Lung ultrasonography to diagnose pneumothorax of the newborn. Am J Emerg Med. 2017;35(9):1298-302. doi: 10.1016/j.ajem.2017.04.001. [PubMed: 28404216].

22. Alrajhi K, Woo MY, Vaillancourt C. Test characteristics of ultrasonography for the detection of pneumothorax: A systematic review and meta-analysis. Chest. 2012;141(3):703-8. doi: 10.1378/chest.11-0131. [PubMed: 21868468].

23. Liu J, Qiu RX, Ren XL, Li JJ, Xia RM, Chi JH. The differentiation diagnosis of respiratory distress syndrome and transient tachypnea of the newborn by lung ultrasound. Chest. 2019;155(4):232A. doi: 10.1016/j.chest.2019.02.221.

24. Ren XL, Fu W, Liu J, Liu Y, Xia RM. Lung ultrasonography to diagnose pulmonary hemorrhage of the newborn. J Matern Fetal Neonatal Med. 2017;30(21):2601-6. doi: 10.1080/14767058.2016.1256997. [PubMed: 27809611].

25. Liu J, Chen XX, Li XW, Chen SW, Wang Y, Fu W. Lung ultrasonography to diagnose transient tachypnea of the newborn. Chest. 2016;149(5):1269-75. doi: 10.1016/j.chest.2015.12.024. [PubMed: 26836942].

26. Liu J, Chen SW, Liu F, Li QP, Kong XY, Feng ZC. The diagnosis of neonatal pulmonary atelectasis using lung ultrasonography. Chest. 2015;147(4):1013-9. doi: 10.1378/chest.14-1306. [PubMed: 25341049].

27. Liu J, Cao HY, Wang HW, Kong XY. The role of lung ultrasound in diagnosis of respiratory distress syndrome in newborn infants. Iran JPediatr. 2015;25(1). e323. doi: 10.5812/ijp.323. [PubMed: 26199698]. [PubMed Central: PMC4505980].

28. Liu J, Liu F, Liu Y, Wang HW, Feng Z. Lung ultrasonography for the diagnosis of severe neonatal pneumonia. Chest. 2014;146(2):383-8. doi: 10.1378/chest.13-2852.

29. Liu J, Chen SW, Liu F, Wang Y, Kong XY, Li QP, et al. BPD, not BPD, or iatrogenic BPD: Findings of lung ultrasound examinations. Medicine (Baltimore). 2014;93(23). e133. doi: 10.1097/MD.0000000000000133. [PubMed: 25415666]. [PubMed Central: PMC4616337]. 